\title{
Farewell, FATZO: a NASH mouse update
}

\author{
Ellen P. Neff
}

T he FATZO mouse ${ }^{1}$ has a new home, and a new name. Originally developed by researchers with preclinical model provider Crown Bioscience, the FATZO strain will now be known as MS-NASH. The change is in conjunction with a new distribution agreement with The Jackson Laboratory, the non-profit rodent model developer and distributor based in Bar Harbor, Maine. The agreement was announced in March 2019, and the Jackson Laboratory has spent the past year establishing a MS-NASH colony at its Sacramento, California breeding facility. They are planning a validation project as well.

'NASH' in the new name refers to 'Non-alcoholic steatohepatitis,' a chronic disease characterized by inflammation and damage to the liver that can lead to cirrhosis, hepatocarcinoma, and liver failure. Along with its precursor, non-alcoholic fatty liver disease (NAFLD), NASH is an emerging epidemic, linked to rising rates of obesity and metabolic disease. A 2015 report $^{2}$ estimates that in the United States alone, between 80 and 100 million adults have NAFLD; $20 \%$ of those will develop NASH, which can be fatal. Currently, there are no approved treatments for the disease, though a number of pharmaceutical companies have NASH drugs in their pipelines ${ }^{3}$.

NASH is a complicated, multifactor disease without clear genetic underpinnings in humans, which makes it difficult to model in mice. "One of the big challenges is to find a mouse model that develops obesity and metabolic disease for the same reasons that people do," says Andrew Schile, a senior scientific advisor who oversees several of the Jackson Laboratory's mouse colonies. "In other words, we want to develop models that include genetic and environmental influences." The hallmarks of NASH include inflammation due to liver damage, fibrosis, and a pathology called 'hepatocellular ballooning.' An 'ideal' mouse model, he says, will develop liver disease along with obesity, high cholesterol, and insulin resistance.

Diet can take many mice a long way towards developing the fatty livers that characterize NAFLD, but diet-induced obese (DIO) mice often won't progress to the more severe signs associated with $\mathrm{NASH}^{4}$. Chemicals, such as streptozotocin

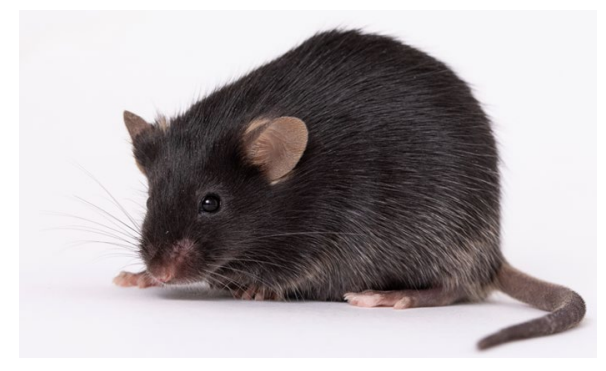

The MS-NASH mouse. Credit: Jennifer L. Torrance, The Jackson Laboratory

(STZ) and carbon-tetrachloride (CCl4), can lead to pathologies such as liver fibrosis, but it's a more artificial process that doesn't reflect the progression of the disease in people. "You and I aren't sucking down carbon tetrachloride and that's why we get fibrosis in our liver, right?" says Megan MacBride, director of commercial models at Taconic Biosciences, a model provider based in Rensselaer, New York. Taconic currently provides an off-the-shelf DIO C57BL/6 model that develops fatty liver but not NASH symptoms-at least through 20 weeks on the current $60 \mathrm{kcal} \%$ high fat diet. The company is looking at older ages, as well as whether additional 'second hit' perturbations might lead to signs of inflammation and fibrosis in the commercially available DIO model. They are also considering a new diet-induced model based on a modified form of the AMLN diet, a ratio of $20 \mathrm{kcal} \%$ fructose, $2 \%$ cholesterol, and $40 \%$ fat, $18 \%$ of which came from trans fats ${ }^{5}$. It takes a bit longer and there can be variability animal-toanimal to contend with, but mice on the original diet develop signs of NASH. However, trans fats have since been banned by the Food \& Drug Administration; Taconic and collaborators are currently evaluating a switch to palm oil in the diet. If validated, they will provide mice at various ages off-the-shelf as well, MacBride says.

The MS-NASH mouse meanwhile is a recombinant inbred cross of two strains: $\mathrm{C} 57 \mathrm{BL} / 6 \mathrm{~J}$ and $\mathrm{AKR} / \mathrm{J}^{1}$. Each is prone to metabolic disease; crossed mice were then selectively bred for enhanced symptoms. Mice will become obese and insulin resistance even on standard chow, but the effects are exaggerated on a high-fat, high-cholesterol diet with fructose-spiked drinking water ${ }^{6}$, says Schile. Markers of liver damage become more pronounced, particularly in males, and the animals will show signs of both hepatocellular ballooning and fibrosis. The Jackson Laboratory is also exploring how immunodeficient, humanized animals, such as the NSG-PiZ strain, might contribute to NASH/NAFLD research, he says; such animals could be transplanted with human liver cells from patients with the disease.

One size will likely not fit all, especially for drug discovery and testing. "When I talk to researchers in this area, they use a number of models," says MacBride, depending on the symptoms and outcome they are looking for in the animals. In their review of NASH models published in November 2017 in Drug Discovery Today, Henrik Hansen et al. conclude: "The ideal model of NASH should faithfully replicate the multifactorial disease mechanisms, while also being reproducible and efficient. Regardless of the approaches currently used to mimic NASH in mice, none of the present models fulfill all requirements for an ideal model. Therefore, selection of the relevant NASH model must be based on prior knowledge of the individual drug target, and it is recommended that at least two individual NASH models should be used for the preclinical characterization of anti-NASH drugs."

Demand for options is there, and on the rise. "We've noticed that there is a growing demand for these models because there's such an unmet need in the community to have good translational models," says Schile.

\section{Ellen P. Neff}

Lab Animal, New York, NY, USA.

e-mail:ellen.neff@us.nature.com

Published online: 6 May 2019

https://oi.org/10.1038/s41684-019-0311-0

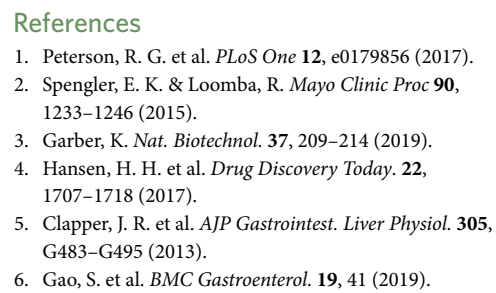

References

Peterson, R. G. et al. PLoS

Spengler, E. K. \& Loomba, R. Mayo Clinic Proc 90

1707-1718 (2017)

G483-G495 (2013).

6. Gao, S. et al. BMC Gastroenterol. 19, 41 (2019). 